\title{
The blackbody radiation in a $D$-dimensional universes
}

$A$ radiação de corpo negro em um universos $\mathrm{D}$-dimensional

\author{
Tatiana R. Cardoso and Antonio S. de Castro ${ }^{1}$ \\ Departamento de Física e Química, Universidade Estudual Paulista 'Júlio de Mesquita Filho', \\ Campus de Guaratinguetá, Guaratinguetá, SP, Brasil \\ Recebido em 30/05/2005; Aceito em 26/09/2005
}

\begin{abstract}
The blackbody radiation is analyzed in a universe with $D$ spatial dimensions. Using the classical electrodynamics suited to the a D-dimensional universe and employing hyperspherical coordinates, the spectral energy density as well as the total energy density are calculated as function of the dimensionality of the universe. The Wien's displacement law and the Stefan-Boltzmann law are properly generalized.
\end{abstract}

Keywords: blackbody, $D$-dimensions, extra dimensions, hyperspherical coordinates

A radiação de corpo negro é analisada em um universo com $D$ dimensões espaciais. Usando a eletrodinâmica clássica consonante com a dimensionalidade do universo, e empregando o sistema de coordenadas hiperesféricas, tanto a densidade de energia espectral quanto a densidade de energia total são calculadas em função do número de dimensões espaciais. A lei do deslocamento de Wien e a lei de Stefan-Boltzmann são apropriadamente generalizadas.

Palavras-chave: corpo negro, $D$ dimensões, dimensões extras, coordenadas hiperféricas

\section{Introduction}

The space dimensionality can be specified by the number $D$, where $D+1$ is the maximum number of points which are equidistant from each other. The dimensionality of the space in which we live, the physical space, is not derived from any physical law but we perceive that we can move ourselves in three distinct directions: to the left and to the right, forward and backward, upward and downward. Therefore, it is convenient for most of the purposes to take for granted that we live in a three-dimensional universe. Obviously this perception of dimensionality is conditioned by the limitations of our senses, and a possible world with more than three dimensions would be properly detected by means of experiments realized in laboratories.

A number of theories involving the unification of the fundamental physical interactions, such as KaluzaKlein [1] and superstring theories [2]-[3], demand physical universes encompassing extra spatial dimensions. It is argued that those extra dimensions do not manifest themselves neither to our sensory experiences nor to the laboratory experiments because such extra dimensions are curled up into dimensions of the order of $10^{-33} \mathrm{~cm}$, an extremely small size to be perceived by our senses

\footnotetext{
${ }^{1}$ E-mail: castro@pesquisador.cnpq.br.

Copyright by the Sociedade Brasileira de Física. Printed in Brazil.
}

or even to be detected by experiments. Physical theories in lower dimensions are also interesting not only as simpler models for the "realistic" three-dimensional world but also for exhibiting some peculiarities that make them different from the 3-dimensional physical theories [4]-[5].

The growing interest in the physics of multidimensional universes calls for a pedagogical approach of a few topics of physics that are usually explored only in the three-dimensional space. The classical electrodynamics in a universe with one and two spatial dimensions has already received a didactic treatment by Lapidus [6]. The author also speculated about the classical electrodynamics in a $D$-dimensional universe. Inspired by Lapidus's work [6], the present paper analyzes the influence of the space dimensionality in the blackbody radiation, one of the first topics the students face at their beginning studies on quantum theory.

The present analysis of the blackbody radiation in a $D$-dimensional universe follows the standard procedure for counting the number of standing wave modes in a cavity (see, e.g., [7]-[8]). Nevertheless, the classical electrodynamics consonant with the dimensionality of the universe in question is used. As a sake of com- 
pleteness, a few criticisms to the literature are also presented. By employing the hyperspherical coordinates, it is shown that both the spectral energy density and the total energy density are dependent on the dimensionality of the universe. Wien's displacement law as well as the Stefan-Boltzmann law are properly generalized. Finally, the readers are instigated to generalize the results for the specific heat of a crystalline solid and the mean particle energy of the ideal Bose gas in a $D$-dimensional universe.

\section{Caveats}

Lapidus [6] argues that the electric field in a onedimensional universe obeys the wave equation. That statement induces unwary readers to guess about the propagation of electric waves in such a universe. Truly speaking, neither there are electric waves nor electromagnetic waves. In such one-dimensional universe the magnetic field is absent and "Maxwell equations" are given by [6]

$$
\frac{\partial E}{\partial x}=\frac{\rho}{\varepsilon_{0}}, \quad \frac{\partial E}{\partial t}=-\frac{J}{\varepsilon_{0}}
$$

where $\varepsilon_{0}$ is the permittivity of free space. The charge and current densities satisfy the equation of continuity: $\partial \rho / \partial t+\partial J / \partial x=0$. In the absence of sources the only possible configuration for the electric field $E$ is to be homogeneous and static. A homogeneous and static electric field always satisfies the wave equation in a trivial way but it is obvious that there is no electromagnetic wave in a one-dimensional universe. The absence of magnetic fields in such one-dimensional universe reinforces our argument. This remark naturally restricts the considerations of the cavity radiation for universes with $D>1$. In order to calculate the electromagnetic modes confined in a cubic cavity, the authors of Refs. [7]-[8], for pedagogical purposes, consider first the modes in a one-dimensional cavity. We emphasize, however, that the calculation refers to the one-dimensional cavity embedded in the three-dimensional world and not a cavity in the one-dimensional world. This argument is strengthen by noting that the authors consider two possible directions for the polarization of the electric field. This observation refuses the statement of the authors of Ref. [8], when they remark that the number of modes in one-dimensional and three-dimensional cavities depend on different powers for frequencies just because the dimensionality of the corresponding universes are different.

The above observations illustrate the subtle and crucial role of the dimensionality of the universe concerning the propagation of electromagnetic waves.

\footnotetext{
${ }^{2}$ Square in the case $D=2$, cube in the case $D=3$.

${ }^{3}$ Circles in the case $D=2$, spherical surfaces in the case $D=3$.
}

\section{Cavity radiation in a $D$-dimensional universe}

The blackbody radiation law is customarily formulated in terms of the electromagnetic energy density inside a cavity into the frequency range between $\nu$ and $\nu+d \nu$. Thus, one must know the density of states in the interval $d \nu$ and multiply it by the average energy.

Let us consider the electromagnetic radiation in thermal equilibrium in a metallic cavity with the shape of a $D$-dimensional cube (hypercube ${ }^{2}$ ) whose walls are maintained at the absolute temperature $T$. Choosing a system of orthogonal Cartesian coordinates with origin at one of the vertices of the cube and axes parallel to their edges, one can write the components of the electric field corresponding to the standing waves in the cavity as

$$
E_{a}\left(x_{a}, t\right)=E_{a}^{(0)} \sin \left(\left|\kappa_{a}\right| x_{a}\right) \sin (2 \pi \nu t)
$$

where $E_{a}^{(0)}$ is the amplitude of the $a$-th component of the electric field oscillating with frequency $\nu, \kappa_{a}$ is the component of the wave number, and $a=1,2, \ldots D$. The electric field must vanish at the walls of the cavity so that the components of the wave number satisfy the conditions

$$
\left|\kappa_{a}\right|=\frac{\pi}{L} n_{a}, \quad n_{a}=1,2,3, \ldots
$$

where $L$ is the length of the edge of the cube. Therefore, the number of possible modes for the electric field in a given state of polarization, into the interval between $\kappa_{a}$ and $\kappa_{a}+d \kappa_{a}$, is given by

$$
N_{P}\left(\kappa_{a}\right) d \kappa_{a}=\frac{L}{2 \pi} d \kappa_{a} .
$$

The presence of the factor 2 in the denominator of (4) allows us to consider that the components of the vector wave number have positive as well as negative values.

The total number of modes in the cavity is the product of the possible modes in each Cartesian axis:

$$
N_{P}(|\vec{\kappa}|) d|\vec{\kappa}|=\prod_{a=1}^{D} N_{P}\left(\kappa_{a}\right) d \kappa_{a}=V \frac{d V_{\kappa}}{(2 \pi)^{D}}
$$

where $V=L^{D}$ is the volume of the cavity and $d V_{\kappa}$ is the infinitesimal $D$-dimensional element of volume in the $\kappa$-space, viz.

$$
d V_{\kappa}=\prod_{a=1}^{D} d \kappa_{a}
$$

This element of volume is, as a matter of fact, a volume which fills the space between the $(D-1)$-dimensional hyperspherical shells ${ }^{3}$ centered about the origin with radii $|\vec{\kappa}|$ and $|\vec{\kappa}|+d|\vec{\kappa}|$. 
In a $D$-dimensional world the electric field has $D$ components and the magnetic field has $D(D-1) / 2$ components [6]. Since the electromagnetic waves are transverse, one can conclude that the electric field has $D-1$ mutually perpendicular components standing at right angles to the direction of the wave propagation. In other words, there are $D-1$ possible directions of polarizations for the electric field ${ }^{4}$. In this way, the number of possible modes, including all possible polarizations of the electric field, is

$$
N(|\vec{\kappa}|) d|\vec{\kappa}|=(D-1) V \frac{d V_{\kappa}}{(2 \pi)^{D}}
$$

To evaluate $d V_{\kappa}$ it is convenient to consider to the hyperspherical coordinates. The hyperspherical coordinates in a space with $D$ dimensions $(D>1)$ are related to the cartesian coordinates by [9]:

$$
\begin{aligned}
x_{1}= & r \cos \theta_{1} \sin \theta_{2} \ldots \sin \theta_{D-1} \\
x_{2}= & r \sin \theta_{1} \sin \theta_{2} \ldots \sin \theta_{D-1} \\
x_{a}= & r \cos \theta_{a-1} \sin \theta_{a} \ldots \sin \theta_{D-1}, \\
& \text { for } 3 \leq a \leq D-1 \\
x_{D}= & r \cos \theta_{D-1},
\end{aligned}
$$

in such a way that the distance of a point to the origin is expressed in terms of the Cartesian coordinates as $r=\sqrt{\sum_{a=1}^{a=D} x_{a}^{2}}$. In addition,

$$
\begin{aligned}
& 0 \leq \theta_{1} \leq 2 \pi, \quad \text { and } 0 \leq \theta_{a} \leq \pi \\
& \text { for } \quad 2 \leq a \leq D-1 .
\end{aligned}
$$

The $D$-dimensional element of volume ${ }^{5}$ and the $(D-1)$ dimensional element of solid angle ${ }^{6}$ are given by

$$
\begin{array}{r}
\prod_{a=1}^{D} d x_{a}=r^{D-1} d r d \Omega, \\
d \Omega=\prod_{a=1}^{D-1}\left(\sin \theta_{a}\right)^{a-1} d \theta_{a} .
\end{array}
$$

For calculating the total solid angle, instead of proceeding with the calculation of the integrals by brute force, some special functions are used. The beta function, $B(z, w)$, can be defined as [10]

$$
\begin{gathered}
B(z, w)=2 \int_{0}^{\pi / 2}(\sin \theta)^{2 z-1}(\cos \theta)^{2 w-1} d \theta \\
=\frac{\Gamma(z) \Gamma(w)}{\Gamma(z+w)}, \quad \operatorname{Re}(z)>0, \quad \operatorname{Re}(w)>0
\end{gathered}
$$

where $\Gamma(z)$ is the gamma function

$$
\Gamma(z)=\int_{0}^{\infty} y^{z-1} e^{-y} d y .
$$

Then, knowing that $\Gamma(1 / 2)=\sqrt{\pi}$, one can write

$$
\int_{0}^{\pi}\left(\sin \theta_{a}\right)^{a-1} d \theta_{a}=\sqrt{\pi} \frac{\Gamma\left(\frac{a}{2}\right)}{\Gamma\left(\frac{a+1}{2}\right)},
$$

in such a manner that the total solid angle is given by

$$
\Omega=2 \pi^{D / 2} \times\left\{\begin{array}{cl}
1 & , \text { for } D=2 \\
\prod_{a=2}^{a=D-1} \frac{\Gamma\left(\frac{a}{2}\right)}{\Gamma\left(\frac{a+1}{2}\right)} & , \text { for } D \geq 3
\end{array}\right.
$$

Writing $\Omega$ in the compact form

$$
\Omega=\frac{2 \pi^{D / 2}}{\Gamma(D / 2)},
$$

one can finally express $d V_{\kappa}$ as

$$
d V_{\kappa}=\frac{2 \pi^{D / 2}}{\Gamma(D / 2)}|\vec{\kappa}|^{D-1} d|\vec{\kappa}| .
$$

Note that if the signs of $\kappa_{a}$ were restricted only to positive values, the factors 2 and $2^{D}$ in the denominators of (4) and (7), respectively, would be absent. In this circumstance only the infinitesimal element of volume in the space $\kappa$ corresponding to $\kappa_{a}>0$ should be considered, implying that the result expressed by (16) should be divided by $2^{D}$ for restrict itself to a $2^{D}$-ant ${ }^{7}$.

Since $|\vec{\kappa}|=2 \pi \nu / c$, where $c$ is the speed of the electromagnetic wave, the number of modes with frequencies between $\nu$ and $\nu+d \nu$ can be written as

$$
N(\nu) d \nu=(D-1) V \frac{2}{\Gamma(D / 2)} \quad \frac{\sqrt{\pi}}{c} \quad \nu^{D-1} d \nu .
$$

Now, each mode has an average energy given by Planck's radiation formula independently of the dimensionality of the universe [7]-[8], i.e.,

$$
\bar{E}=\frac{h \nu}{\exp \left(h \nu / k_{B} T\right)-1},
$$

where $h$ and $k_{B}$ are the Planck and Boltzmann constants, respectively, in such a way that the energy den$\operatorname{sity}^{8}$ within the interval between $\nu$ and $\nu+d \nu$ is given by

$$
\begin{gathered}
\rho_{T}(\nu) d \nu=2\left(\frac{\sqrt{\pi}}{c}\right)^{D} \frac{D-1}{\Gamma(D / 2)} \times \\
\frac{h \nu^{D}}{\exp \left(h \nu / k_{B} T\right)-1} d \nu .
\end{gathered}
$$

\footnotetext{
${ }^{4}$ If the remarks in Section 2 were not considered, one might conclude that there are no electromagnetic waves in an one-dimensional universe but there might be longitudinal electric waves. This last possibility, however, only can be excluded by the analysis of Maxwell's equations expressed by (1).

${ }^{5}$ Element of area in the case $D=2$.

${ }^{6}$ Element of plane angle in the case $D=2$.

${ }^{7}$ A quadrant in the case $D=2$, an octant in the case $D=3$, and so on.

${ }^{8}$ Energy per unit area in the case $D=2$, energy per unit volume in the case $D=3$, and so on.
} 
This is the mathematical expression for the Planck blackbody spectrum in a $D$-dimensional universe. It gives the spectral distribution for the energy in the cavity. Fig. 1 illustrates the spectral energy density (energy density per unit frequency) for the particular cases $D=2,3$ and 4 , for $T=1500 \mathrm{~K}^{9}$.

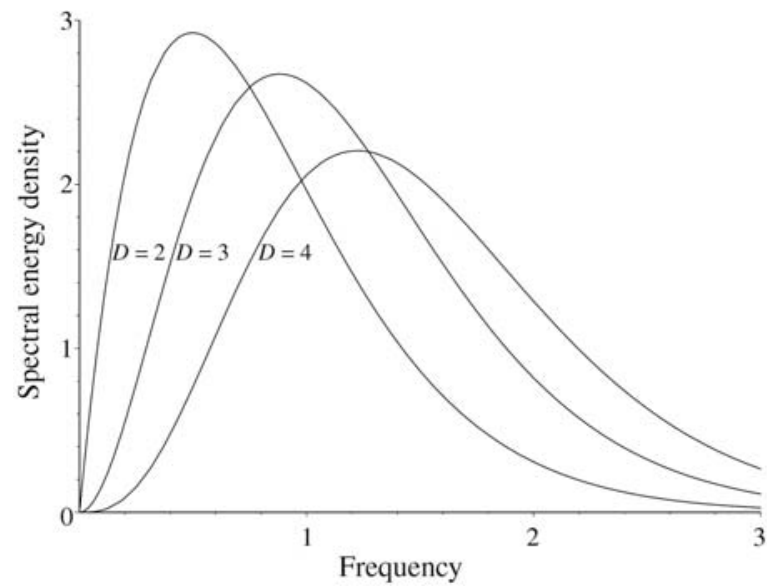

Figure 1 - Spectral energy density as a function of $\nu$ for $T=1500 \mathrm{~K}$. Using the physical constants in SI, the frequency must be multiplied by $10^{14}$ in order to be expressed in $\mathrm{Hz}$, whereas the spectral energy density must be multiplied by $10^{-23}$, $10^{-17}$ and $10^{-11}$ in order to have the units $\mathrm{W} \cdot \mathrm{m}^{-1} \cdot \mathrm{Hz}^{-1}$, $\mathrm{W} \cdot \mathrm{m}^{-2} \cdot \mathrm{Hz}^{-1}$ and $\mathrm{W} \cdot \mathrm{m}^{-3} \cdot \mathrm{Hz}^{-1}$, in the cases $D=2,3$ and 4 , respectively.

The result known as Wien's displacement law, relating the frequency, where the the spectrum reaches a maximum, with the temperature, i.e. $\nu_{\max } / T=$ $\alpha=$ const, is valid independently of the universe dimensionality. Nevertheless, the constant $\alpha$ increases as the number of the dimensions of the universe increases. Indeed, substituting $x=h \nu_{\max } / k_{B} T$ in (19) and making $d \rho_{T}(x) / d x=0$, one finds

$$
e^{-x}=1-\frac{x}{D}
$$

Eq. (20) has as positive solution ${ }^{10}$

$$
x=D+W\left(-D e^{-D}\right),
$$

where $W$ is the Lambert function defined as [11]

$$
W(z)+e^{W(z)}=z .
$$

Note that if $x$ is a function of the space dimensionality for $D>1$, it is a monotonic increasing function. This happens because the second term at the right hand side of (21) is a monotonic increasing function for $D>1$ and tends asymptotically to zero as $D \rightarrow \infty$. Of course, the very same conclusion concerning the behavior of $\alpha$ as a function of $D$ could be obtained in a simpler manner by plotting Eq. (20). It should be mentioned, though, that the numerical solution of (20) becomes extremely easy by using computer algebra softwares which contains the Lambert $W$ function.

The total energy density in the cavity is obtained by integrating the spectral energy density over all frequencies, $\rho_{T}=\int_{0}^{\infty} \rho_{T}(\nu) d \nu$, resulting in

$$
\rho_{T}=a_{D} T^{D+1} \text {. }
$$

Using the identities [10]

and

$$
\Gamma(z+1)=z \Gamma(z)
$$

$$
\Gamma(2 z)=\frac{2^{2 z-1 / 2}}{\sqrt{2 \pi}} \Gamma(z) \Gamma\left(z+\frac{1}{2}\right),
$$

the constant of proportionality $a_{D}$ can be written as

$$
\begin{aligned}
a_{D}= & \left(\frac{2}{h c}\right)^{D}(\sqrt{\pi})^{D-1} k_{B}^{D+1} D(D-1) \times \\
& \Gamma\left(\frac{D+1}{2}\right) \zeta(D+1),
\end{aligned}
$$

where $\zeta(z)$ is the Riemann zeta function [10]:

$$
\zeta(z)=\sum_{n=1}^{\infty} n^{-z}, \quad \operatorname{Re}(z)>1,
$$

which can also be written in the form

$$
\zeta(z)=\frac{1}{\Gamma(z)} \int_{0}^{\infty} \frac{y^{z-1}}{e^{y}-1} d y .
$$

The radiation energy rate per unit area, known as the radiancy, is defined by the total energy emitted per unit time and per unit area of the cavity surface. The radiancy and the energy density in the cavity are related by purely geometric factors. It results that such quantities are proportional to each other, as one will see.

The energy emitted by an infinitesimal element of area $d A$ of the surface of the cavity in the interval of time $d t$ occupies a hemisphere $(D-1)$-dimensional with radius $c d t$ centered about $d A$. The energy contained in a cylinder ${ }^{11}$ of length $c d t$, with inclination $\theta_{D-1}$ with respect to the Cartesian axis $x_{D}$ perpendicular to the element of area, is

$$
d U_{T}=\rho_{T} c d t d A \cos \theta_{D-1} .
$$

Nevertheless, only the fraction $d \Omega / \Omega$ of the energy contained in the cylinder propagates in the direction specified by the angle $\theta_{D-1}$. Therefore, the energy emitted in the direction specified by the angle $\theta_{D-1}$ is

$$
R_{T}\left(\theta_{D-1}\right)=\rho_{T} c \frac{d \Omega}{\Omega} \cos \theta_{D-1} .
$$

\footnotetext{
${ }^{9}$ Due to errors on the scales of the abscissa and ordinate axes in the figures 1-1 and 1-8 in the Ref. [8], the comparison with the Fig. 1 can not be done.

${ }^{10} x=0$ is another possible solution.

${ }^{11}$ In the case $D=2$ the cylinder is a rectangle, the element of area is an element of line and the hemisphere is a semicircle.
} 
In order to obtain the energy emitted all over possible directions one must integrate the previous expression over all angles that sweep the hemisphere. The results

$$
R_{T}=\frac{\rho_{T} c}{\Omega} \times\left\{\begin{array}{ccc}
2 & \text { for } & D=2 \\
\pi & \text { for } & D=3 \\
\theta & \text { for } & D \geq 4
\end{array}\right.
$$

where

$$
\theta=2 \frac{(\sqrt{\pi})^{D-1}}{D-1} \prod_{a=2}^{a=D-2} \frac{\Gamma\left(\frac{a}{2}\right)}{\Gamma\left(\frac{a+1}{2}\right)},
$$

can even be written as

$$
R_{T}=\frac{(\sqrt{\pi})^{D-1}}{\Gamma\left(\frac{D+1}{2}\right)} \frac{\rho_{T} c}{\Omega}=\frac{\Gamma\left(\frac{D}{2}\right)}{\Gamma\left(\frac{D+1}{2}\right)} \frac{c}{2 \sqrt{\pi}} \rho_{T} .
$$

Now we are ready to establish the law which relates the radiancy with the temperature, the generalized Stefan-Boltzmann law:

$$
R_{T}=\sigma_{D} T^{D+1},
$$

where the factor $\sigma_{D}$ is the generalized StefanBoltzmann constant, given by

$$
\begin{aligned}
\sigma_{D}= & \left(\frac{2}{c}\right)^{D-1}(\sqrt{\pi})^{D-2} \frac{k_{B}^{D+1}}{h^{D}} D(D-1) \times \\
& \Gamma\left(\frac{D}{2}\right) \zeta(D+1) .
\end{aligned}
$$

We conclude that the generalized Stefan-Boltzmann constant is a monotonic increasing function of the number of space dimensions.

\section{Conclusions}

We showed that the use of hyperspherical coordinates allows us to treat analytically the blackbody radiation in a $D$-dimensional universe. The case of onedimensional cavities was excluded from the analysis by taking the possible independent directions for the polarization of an electromagnetic wave into account.

Experimental results are consistent with the blackbody radiation in a three-dimensional world. Since higher dimensional universes with such extra dimensions properly made compact were not considered, the possibility of higher dimensional universes can not be discarded by contrasting the experimental data with the analysis done in this work.

It is worthwhile to observe that the results of this paper would be the same if one had considered the photon gas obeying the usual Bose-Einstein distribution. This is the case because one would had to consider that each photon with energy $h \nu$ can have $D-1$ possible states of polarization.

The formalism developed in this work can be used to generalize other quantities of the quantum theory in an easy way. For instance, the calculation of the vibration modes of atoms in an elastic solid, or the number of states of a phonon gas, for the generalization of the Debye theory of the specific heat of a solid. In this scenario, diversely from the case of electromagnetic waves, longitudinal acoustic waves are feasible in a one-dimensional universe. It is anticipated that the specific heat at high temperatures behaves as $C_{V}=D R$ (the generalized Dulong e Petit law), where $R$ is the universal gas constant, and that at low temperatures it behaves like $C_{V} \sim T^{D}$. Another interesting subject is related to the Bose condensation. In this case the number of quantum states of the ideal gas with energy within the interval $E$ and $E+d E$ is proportional to $E^{D / 2-1} d E$, a result divergent at $E=0$ for $D=1$ and independent of $E$ for $D=2$. The calculations which lead to these results, as well as the calculation of the average particle energy of the ideal Bose gas (with a puzzle in the calculation of the number of particles for $D<3$ ), are left to the readers.

\section{Note added in proof}

After the paper was submitted, we become aware of the more technical and quasi-homonymous "Blackbody radiation in extra dimensions" by $\mathrm{H}$. Alnes, F. Ravndal and K. Wehus (arXiv: quant-ph/0506131), uploaded to the archives seventeen days later.

\section{Acknowledgments}

This work was supported in part funding agencies CNPq and FAPESP.

\section{References}

[1] R. Coquereaux and A. Jadezyk, Riemannian Geometry, Fiber Bundles, Kaluza-Klein Theories and All That... (World Scientific, Singapore, 1988).

[2] M. Green, J. Schwarz and E. Witten, Superstring Theory (Cambridge University Press, Cambridge, 1987).

[3] V.O. Rivelles, Ciência Hoje 23, 46 (1998).

[4] E. Abdalla, M.C.B. Abdalla and K.D. Rothe, NonPerturbative Methods in 2-Dimensional Quantum Field Theory (World Scientific, Singapore, 1991).

[5] C.J. Efthimiou and D.A. Spector, A Collection of Exercises in Two-Dimensional Physics, Part. I, arXiv: hepth/0003190.

[6] I.R. Lapidus, Am. J. Phys. 50, 155 (1982).

[7] R.P. Feynman, R.H. Leighton and M. Sands, The Feynman Lectures on Physics (Addison-Wesley, Reading MA, 1964), v. I and III.

[8] R. Eisberg and R. Resnick, Quantum Physics of Atoms, Molecules, Solids, Nuclei, and Particles (Wiley, New York, 1974).

[9] A. Erdelyi, Higher Transcendental Functions (McGraw-Hill, New York, 1953), v. 2.

[10] M. Abramowitz and I. A. Stegun, Handbook of Mathematical Functions (Dover, Toronto, 1965).

[11] R.M. Corless, G.H. Gonnet, D.E.G. Hare, D.J. Jeffrey and D.E. Knut, Adv. in Comp. Math. 5, 329 (1996). 